\section{Mit mehr Markt zur Nachhaltigkeit}

\author{
Mit der Betonung der grundsäłzlichen Gleichrangigkeit von Ökonomie, Ökologie \\ und Sozialem wird aus einem zunächst primär ökologisch ausgerichteten ein \\ gesellschaftspolitisches Nachhaltigkeitspostulat. Dieses sollte aber nicht als \\ umfangreicher Planungsansatz verstanden werden, vielmehr sollte dem \\ Steuerungssystem Markt grundlegende Bedeutung zukommen.
}

$\mathrm{E}$ Von Paul Klemmer ine befriedigende Nachhaltigkeitspolitik muß unbedingt dem Tatbestand Rechnung tragen, daß der Wunsch nach langfristig durchhaltbarer Entwicklung nicht nur durch ökologische, sondern auch durch ökonomische und soziale Risiken bedroht werden kann. Diese Feststellung findet immer mehr Anerkennung und wurde auch in der Enquete-Kommission von allen Gruppierungen getragen. Diese Interpretation des Nachhaltigkeitsprinzips betont die grundsätzliche Gleichrangigkeit der drei Teilanliegen und versteht sich nicht mehr als Maximierung ökologischer Belange unter Berücksichtigung ökonomischer und sozialer Nebeneffekte. Damit potenzieren sich einerseits die zu bewältigenden Zielfindungs-, Zieloperationalisierungs-, Zielgewichtungs- und Zielumsetzungsprobleme, andererseits schwindet aber auch immer mehr der Unterschied zur klassischen Gesellschaftspolitik, die sich ebenfalls als umfassend und langfristig orientiert verstand.

\section{Konkurrenz zweier Richtungen}

Was die Konkretisierung einer solchen Nachhaltigkeitspolitik betrifft, konkurrierten in der Enquete-Kommission zwei Richtungen miteinander, die im Endbericht immer wieder auf unterschiedliche Weise durchschimmern und auch zu einer gewissen Heterogenität des Abschlußtextes führten. Eine erste Richtung, die man plakativ als planerisch bezeichnen kann, strebt eine Art gesellschaftlichen Nachhaltigkeitsplan an. Sie präferiert hierbei zumeist eine indikative Planung, die die konsensuale Zielbildung auf breiter Basis in den Vordergrund stellt und die Partizipation möglichst vieler gesellschaftlicher Gruppen sowie die Integration betont. Damit möchte man die traditionell additive Rolle der Umweltpolitik überwinden und Ökologie, Ökonomie und Soziales als Einheit ansehen. Gefordert werden in der Regel eine diskursive und konsensuale Zielfindung, spezielle Planungsinstitutionen (etwa einen „Rat für Nachhaltige Entwicklung" mit Vetorecht oder als großen „Mahner"), ein möglichst quantitative Handlungsziele verfolgender Planentwurf als staatliche Vorgabe bezüglich wichtiger ökologischer, ökonomischer und sozialer Belange, eine stark auf ökonomischen Anreizmechanismen beruhende und verhandlungsorientierte Planumsetzung sowie ein ausgefeilter Berichts- und Über-

Dieser Weg zu einem kompatiblen Nachhaltigkeits-Zielsystem ist eindeutig mehr als die Vorgabe mindestens einzuhaltender Bedingungen (Leitplankenkonzept) und geht in die Richtung eines Leitstrahlkonzepts mit beachtlichen Vorgaben, die im Extrem auch einzelne Individuen betreffen können. Hinter dieser Denkrichtung stehen Gruppierungen, die individualistischen Gesellschaftskonzepten (Vorwurf der Kurz- und Engsichtigkeit individueller Entscheidungen) sowie Marktlösungen (angebliche ökologische Blindheit der Märkte) zumeist äußerst skeptisch gegenüberstehen. Sie haben großes Interesse an einer staatlich geleiteten Aufdeckung und Korrektur angeblich verzerrter individueller Präferenzen, wobei unterstellt wird, daß durch Information und gesellschaftlichen Diskurs eine Präferenzenharmonisierung in Richtung auf mehr Nachhaltigkeit erreicht werden kann.

Mehrheitlich sehen die Vertreter dieser ersten Richtung einer Nachhaltigkeitspolitik die hierbei zu bewältigenden Probleme, und zwar zunächst die riesigen Informationsprobleme beim Zielfindungsprozeß, dann aber auch die Zielumsetzungsfragen bei einem tendenziell immer schwächer werdenden Staat, sie halten sie aber für lösbar.

Dieser ersten Richtung einer Nachhaltigkeitspolitik mit spezifischen Handlungszielen für die drei Säulen Ökologie, ökonomie und prïfungsmechanismus.
Soziales steht eine zweite entgegen, die man auch als ordnungspolitischen oder marktwirtschaftlichen Ansatz kennzeichnen kann. Sie bevorzugt einen eher systemtheoretischen Ansatz, der sich vor allem an der Aufrechterhaltung der Funktionsfähigkeit des ökonomischen Steuerungssystems marktwirtschaftlicher Prägung orientiert. Insbesondere wird hervorgehoben, daß wirtschaftliche Systeme viele Gemeinsamkeiten mit ökologischen Systemen haben und ebenfalls überfordert werden können, was einen ökonomischen Kollaps herbeiführen würde. So sind ökologische Systeme durch hohe Komplexität, wechselseitige Verflechtung, häufig nichtlineare Zusammenhänge und hohe Selbststeuerungsfähigkeit, die auch Belastbarkeit und Schockabsorptionsfähigkeit gewährleistet, gekennzeichnet. In gleicher Weise wie das ökologische ist das wirtschaftliche System marktwirtschaftlicher Prägung ein nicht-autoritärer Selbststeuerungsmechanismus, der unter Wettbewerbsbedingungen gesellschaftlich wünschenswerte Leistungen wie Anpassung der Produktion an die Konsumentenwünsche, statische und dynamische Effizienz, hohe Reagibilität auf Datenänderungen, Fähigkeit zur Innovation und Wissensproduktion und (etwa über Preissenkungen) sogar Verteilungsfunktionen erbringt und in dieser Hinsicht, wie die Vergangenheit zeigte, autoritären Systemen weit überlegen ist.

\section{Koordinationsprozeß Markt}

Im marktwirtschaftlichen System erleben die Marktteilnehmer Bestätigungen ihrer Planvorstellungen, aber auch Enttäuschungen oder positive Überraschungen, und lernen aus Erfahrungen sowie Irrtümern. In diesen umfassenden Koordinationsprozeß fließen nicht nur Vergangenheitserfahrungen, sondern - was sehr wichtig ist - auch divergierende Erwartungen über die Zukunft ein. Märkte können somit eine höhere Korrespondenz von Erwartungen und damit eine Ex ante-Koordination bewirken. Dies kann auch die Berücksichtigung von intergenerationellen Verteilungsvorstellungen beinhalten. So ist schon allein die Tatsache, daß Eltern ihren Kindern Vermögen vererben, ein Hinweis darauf, daß die heutige Generation auch an die künftige denkt und zumindest eine Art DreiGenerationen-Planungsperspektive (Berücksichtigung der Enkel) unterstellt werden kann. Je wohlhabender die heutige Generation ist und 
je stabiler die wirtschaftlichen Rahmenbedingungen, insbesondere die.von der Politik gesetzten Rahmenbedingungen sind, desto eher sind die planenden Wirtschaftssubjekte bereit, Langfristüberlegungen einzubeziehen.

Die sich in diesem System unter Wettbewerbsbedingungen herausbildenden Preise sind konzentrierte Informationsvermittler sowie unpersönliche bzw. unpolitische Signale. Sie stellen ein Medium der Wissensvermittlung dar, in die die gesellschaftliche Bewertung - verstanden als individualistisches Gesellschaftskonzept von Gütern, Dienstleistungen sowie von Informationen einfließt.

\section{- Nachhaltiger mit mehr Markt}

Dieses System kann auch einen effizienteren Umgang mit den Umweltfunktionen herbeiführen. Was die hierfür erforderliche stärkere Ankopplung des ökonomischen Systems an die ökologischen Restriktionen betrifft, ist zu beachten, daß Restriktionen vom Markt in der Regel nur in codierter Form wahrgenommen werden. Insofern muß man die Lenkungsund Steuerungsfunktionen dieser Codes - nämlich der relativen Preise - verbessern. Die Setzung dieser Codes ist, was häufig übersehen wird, jedoch nicht Staatsaufgabe, sondern sollte über Märkte vorgenommen werden, was wiederum bedeutet, daß private Eigentumsrechte an Umweltgütern sowie deren Handel zugelassen werden müssen und gravierende externe Effekte vermieden werden sollten.

Vor diesem Hintergrund versteht man, warum der Abschlußbericht der Enquete-Kommission die ökonomische Säule weitgehend über Forderungen definiert, die der Aufrechterhaltung der Leistungsfähigkeit des Markt- und Wettbewerbssystems und der Ausweitung seiner Lenkungsfunktion auf Umweltgüter dienen sollen. Es ist eigentlich nur zu bedauern, daß der hier aufleuchtende Gedanke, mehr Nachhaltigkeit mit und durch mehr Marktwirtschaft zu erreichen, im weiteren Verlauf des Berichts nicht mehr weiter verfolgt wird, sondern sich in weiten Teilen wieder der Wunsch nach mehr Umwelt- bzw. Nachhaltigkeitsplanung in den Vordergrund schiebt bzw. durchsetzt.

Statt dessen hätte man prüfen können, inwieweit mehr Nachhaltigkeit über mehr Marktwirtschaft erreicht werden kann (1) und wo für einzelne Umweltprobleme Marktversagenstatbestände vorliegen oder spezifische normative Anliegen $\mathrm{zu}$ berücksichtigen sind, die eventuell staatliche Eingriffe legitimieren. So können einzelne Umweltfunktionen als Kollektivgüter (z.B. Erdatmosphäre) gekennzeichnet werden, bei denen aufgrund eines defizitären Ausschlußprinzips eine Funktionsminderung oder sogar ein Funktionsảusfall droht, wobei der räumliche Umfang des Betroffenenkreises differiert. Hier wäre zunächst festzulegen, auf welcher kollektiven Ebene Entscheidungen hinsichtlich des Ausmaßes der zur heutigen oder künftigen Nutzung zur Verfügung stehenden Umweltfunktionen getroffen werden müssen, um dann den auf diesen Ebenen definierten regionalen oder globalen Nutzungsspielraum auf die einzelnen Nutzungsinteressierten innerhalb des Kollektivs zu verteilen.

\section{Leitplanken und Rahmensetzung}

Bei solchen Kollektivgütern benötigt der Markt, über den auf Dauer die Zuweisung der individuellen Nutzungsrechte (etwa Handel mit Emissionsrechten) erfolgen soll, Vorgaben eben Grenzziehungen einer maximal zulässigen Nutzung (Leitplankenkonzept). Die Berücksichtigung marktwirtschaftlicher Überlegungen zielt dabei nicht nur auf die Gestaltung des Zuweisungsprozesses der Nutzungsrechte, wo man den Individuen die größtmögliche individuelle Handlungsfreiheit belassen sollte, sondern auch auf die Entscheidungsfindung. Da diese nicht nur auf naturwissenschaftlichen Fundamenten (UrsacheWirkungsforschung), sondern auch auf Kosten- und Nutzenvorstellungen beruht, sollten individuelle Bewertungsaspekte mit berücksichtigt werden.

Dem sich bei marktlicher Koordination ergebenden Problem der Unsicherheit über die Konsequenzen der eigenen Handlungen auch für zukünftige Generationen und der nur unzureichend verfügbaren - weitgehend als öffentliches Gut anzusehenden - Informationen bezüglich der Ursache-Wirkungs-Zusammenhänge ist durch eine ordnungspolitische Rahmensetzung zu begegnen, die vorrangig mit Hilfe eines Ausbaus des Haftungsrechts sowie einer Intensivierung der Konkurrenz zwischen technischen und wissenschaftlichen Sachverständigen wettbewerbliche Anreize zur Informationsermittlung auslöst. Die Grenzen einer Beschränkung auf rein individuelle Entscheidungen zum Umgang mit Risiken sind insbesondere dann erreicht, wenn nicht privatisierbare Elemente von Umweltfunktionen betrof- fen sind oder eine Vertretung der Interessen zukünftiger Generationen, z.B. durch Vorfahren, nicht gewährleistet ist. Hier sind wiederum kollektive Entscheidungsverfahren erforderlich, in denen jedoch Anreize zu einer möglichst umfassenden Nutzung der individuellen Bewertungen zu bilden sind.

Eingriffe in die Preisfindung für solche Umweltfunktionen, für die individuell zuordbare Handlungs- und Verfügungsrechte definiert werden können, um den Präferenzen zukünftiger Generationen Geltung zu verschaffen, können im Rahmen eines ordnungspolitischen Konzepts nur insoweit gerechtfertigt werden, als nachzuweisen ist, daß mit Hilfe dieser Maßnahmen eine intertemporal effizientere Allokation vorgenommen werden kann. Hier gilt aber der alte Erfahrungssatz, daß politische Entscheidungen in der Regel von einem noch kurzfristigeren Bezugszeitraum ausgehen als private Kalküle.

Anreize für die Individuen zu einem langfristigen Kalkül, das auch zukünftige Folgen einbezieht, können insbesondere von einer Verstetigung der praktischen Umwelt- und Wirtschaftspolitik ausgehen. Anstatt dem Staat die Aufgabe zu übertragen, kurzfristig in die Preisfindung einzugreifen, ist durch die Bildung eines verläßlichen und akzeptierten allgemeinen Rahmens, der den Individuen die gesicherte Erstellung eines langfristigen Kalküls ermöglicht, eher von einer echten Knappheitsorientierung bei der Preisfindung auszugehen.

\section{Anmerkung}

(1) Vgl. hierzu Klemmer, Paul, Dorothee Becker-Soest, Rüdiger Wink (Hrsg.): Liberale Grundrisse einer zukunftsfähigen Gesellschaft, Nomos, Baden-Baden 1998.

\section{Der Autor}

Dr. Paul Klemmer ist Professor an der Ruhr-Universität Bochum und Prüsident des Rheinisch-Westfälischen Instituts für Wirtschaftsforschung. Er war als Sachverständiger Mitglied der Enquete-Kommission "Schutz des Menschen und der Umwelt".

Kontakt: An der Pfannenschmiede 9, 45549 Sprockhövel, Tel. $02324 / 971871$, Fax 02324/725 50,

E-mail: Paul.Klemmer@rz.ruhr-uni-bochum.de 
(c) 20I0 Authors; licensee IÖW and oekom verlag. This is an article distributed under the terms of the Creative Commons Attribution Non-Commercial No Derivates License (http://creativecommons.org/licenses/by-nc-nd/3.o/), which permits unrestricted use, distribution, and reproduction in any medium, provided the original work is properly cited. 